\title{
ASSOCIATION OF RIBOFLAVIN, CAFFEINE, AND SODIUM SALICYLATE IN AQUEOUS SOLUTION
}

\author{
S. F. Baranovskii and P. A. Bolotin ${ }^{*}$
}

UDC $577.113+541.49$

We have used $U V$ and visible spectrophotometry to study self-association of aromatic riboflavin molecules (RFN, vitamin $B_{2}, 7,8$-dimethyl-10- $N$-( $I^{\prime}$-D-ribityl)isoalloxazine) in aqueous solution ( $p H$ 6.86) at $T=298 \mathrm{~K}$, using a dimer model. We have determined the equilibrium dimerization constant for riboflavin, $K_{d B}=125 \pm$ $40 \mathrm{M}^{-1}$. We have studied heteroassociation in the system of molecules of 7,8-dimethyl-10-ribitylisoalloxazine with 1,3,7-trimethylxanthine (caffeine) and sodium salicylate (NAS) in aqueous solution ( $p H$ 6.86; $T=298 \mathrm{~K}$ ). We have determined the heteroassociation constants for RFN-NAS and RFN-caffeine molecules in the absence and in the presence of urea in solutions using a modified Benesi-Hildebrand equation: $25 \pm 4,17 \pm 3$, and $74 \pm 11,53 \pm 7 \mathrm{M}^{-1}$ respectively. We have determined the dimerization constants for NAS $\left(2.7 \pm 0.5 \mathrm{M}^{-1}\right)$ and caffeine $\left(17.0 \pm 1.5 \mathrm{M}^{-1}\right)$. We conclude that heteroassociation of the aromatic molecules leads to a lower effective riboflavin concentration in solution, and the presence of urea in mixed solutions leads to an decrease in the complexation constants for the RFN-NAS and RFN-caffeine systems.

Key words: spectrophotometry, riboflavin, caffeine, sodium salicylate, urea, self-association, dimer model, heteroassociation, dimerization constant, heteroassociation constant.

Introduction. Effective anti-tumor chemotherapy calls for simultaneous use of two or more cytostatics. Possible interaction between drugs included in the combination may alter the pharmacokinetic and pharmacodynamic characteristics of the individual components, leading to weakening or conversely enhancement of the anti-tumor effect and the toxicity [1]. Due to lack of clear understanding of the biochemical and molecular mechanisms of interactions between drugs used in combination, the synergism of the biological action of chemotherapeutic drugs has not been satisfactorily explained either at the cellular or the molecular level.

We know [2] that molecules of anthracycline antibiotics in the millimolar concentration range exhibit a strong tendency toward self-association in solution. This is expressed in the formation of dimers and higher order associates, stabilized as a result of interaction between the aromatic chromophores of the molecules in the aggregates (stacking) [2]. Such interactions are also observed with heteroassociation of antibiotics with aromatic biologically active compounds in solution, for example with caffeine [3] and riboflavin (RFN, vitamin $B_{2}$ ) [4]. It is hypothesized [5] that this mechanism dominates in the process of degradation of anthracycline antibiotics, in particular doxorubicin, and some aromatic carcinogens when bound to riboflavin. A protective effect of riboflavin relative to DNA was observed earlier in $[6,7]$, expressed in the significantly decreased binding of aromatic mutagens with nucleic acid in the presence of the vitamin. Furthermore, it was shown that other aromatic compounds such as caffeine can intercept antibiotic and mutagen molecules by means of heteroassociation and compete with them for DNA binding sites [8].

In this paper, we study the complexation of molecules of organic compounds: riboflavin, sodium salicylate (NAS, an analog of aspirin), and caffeine in the presence and in the absence of urea, which may enter the human body as food ingredients and as medicinal drugs. Their biological activity may change considerably when they enter the cell in combination [9].

Flavin molecules are based on an isoalloxazine ring (Fig. 1a), which is pteridine condensed with a benzene ring. The flavin structure has four nitrogen hetero atoms, methyl substituents at the 7 and 8 positions of the benzene

*To whom correspondence should be addressed.

Sevastopol National Technical University, Studgorodok, Sevastopol 99053, Ukraine. E-mail: piterbol@inbox.ru. Translated from Zhurnal Prikladnoi Spektroskopii, Vol. 74, No. 2, pp. 188-194, March-April, 2007. Original article submitted October 25, 2006. 

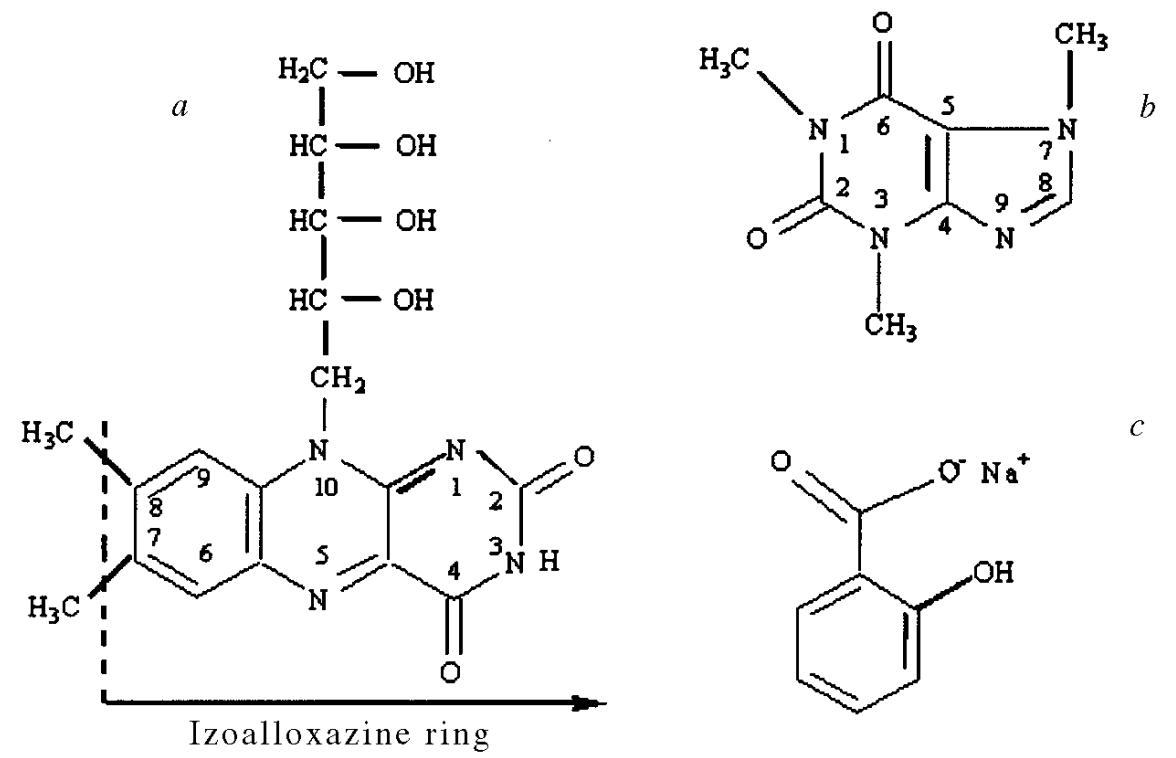

Fig. 1 Structural formulas for the riboflavin (a), caffeine (b), and sodium salicylate (c) molecules.

ring, and also a substituent added to the heterocyclic nitrogen atom at the 10 position. For the flavin widely distributed in nature, riboflavin or ribitylflavin (7,8-dimethyl-10-N-(1'-D-ribityl)isoalloxazine), the latter substituent is the sugar alcohol ribitol. This highly hydroxylated substituent is rather labile and plays the role of a linker in formation of coenzymes. The chemical structure of riboflavin is highly specific, since even slight modifications of the isoalloxazine ring and the ribityl residue in the molecule has a strong effect on the catalytic properties of the enzyme, loss of vitamin activity, or formation of antagonists [10].

Free riboflavin is readily reduced, and its reduced form is readily oxidized again. This property is used in the biological function of flavin coenzymes in the electron transport chain.

Riboflavin has photochemical activity, is known as a typical ${ }^{1} \mathrm{O}_{2}$-sensitizing factor [11], and can be used to inactivate contaminated medical samples such as blood plasma [12,13]. Riboflavin deficiency causes formation of individual breaks in the helix of nuclear DNA [14], increases binding of carcinogens to DNA [7], and decreases the glutathione content in the liver [15].

Caffeine (1,3,7-trimethylxanthine), a methylated purine derivative, is in the methylxanthine class. Caffeine molecules (Fig. 1b) are planar, electrically neutral heterocyclic compounds containing methyl groups at the 1, 3, 7 positions, oxygen at the 2,6 positions, and four nitrogen hetero atoms. The biological activity of caffeine is exhibited in its ability to decrease the carcinogenic effects of mutagenic drugs [16]. It has been established that caffeine can be used as a prophylactic anticarcinogen when there is risk of developing cancer of the digestive tract [17-19], breast cancer [20,21], pancreatic cancer [22], and thyroid cancer [23]. According to modern data, an important role in the mechanism of action for caffeine is played by its inhibiting effect on the enzyme phosphodiesterase, which leads to intracellular accumulation of cyclic adenosine monophosphate.

Sodium salicylate (NAS), the sodium ester of salicylic acid (Fig 1c), is a nonsteroidal anti-inflammatory drug containing a carboxyl anion in the 1 position and a hydroxyl group in the 2 position of the benzene ring, which makes it quite soluble in water. NAS has a pharmacological action similar to that of acetylsalicylic acid (aspirin).

Urea, $\mathrm{H}_{2} \mathrm{~N}-\mathrm{CO}-\mathrm{NH}_{2}$ (carbamide, the end product of protein metabolism in vertebrates) is widely used as a denaturant. In aqueous solutions in concentrations 6-8 M, urea breaks down the tertiary structure of proteins [24]. The molecular mechanism of action of urea, which is the basis for the change in the natural properties of proteins, is still unclear [25]. In [26], urea is considered as a "structure breaker" for water, which interferes with the ability of water molecules to maintain a tetrahedral geometry of the hydrogen bonds and weakens the hydrophobic effect upon association of the molecules. 
Salicylates of alkali metals, urea, and alkaloids like caffeine, along with their therapeutic properties have hydrotropic properties [27, 28], as is apparent in their ability to increase many-fold the solubility of therapeutic drugs [2932] including riboflavin [33]. Mechanisms underlying their hydrotropic action include specific interaction with the solvent, self-association of the hydrotropic agent, and complexation between the hydrotropic and drug molecules [34-36].

The aim of this work was to determine the ability of biologically active NAS and caffeine molecules to form noncovalent complexes with riboflavin, and the possibility of regulation of the pharmacological activity when they are used in combination. We have used optical spectrophotometry to study self-association of riboflavin molecules in aqueous solution. We studied heteroassociation of the molecular systems RFN-NAS and RFN-caffeine in the absence and in the presence of urea. Quantitative analysis was performed using the experimental concentration dependences of the optical density at the wavelength of the riboflavin monomer absorption maximum.

Experimental Procedure. The electronic absorption spectra were recorded on an SF-46 spectrophotometer in the visible region. In the self-association study, the optical density of the riboflavin solutions were measured in the wavelength range 340-520 nm. Complexation of mixed solutions of RFN-NAS, RFN-caffeine, RFN-NAS-urea, and RFN-caffeine-urea were studied in the wavelength range 390-520 nm.

Riboflavin from Serva Feinbiochemica GmbH (Heidelberg, Germany), sodium salicylate, caffeine, and urea from Sigma (USA) were used with no additional purification. The starting solutions of the studied drugs were obtained by addition of a weighed amount of the sample in $0.1 \mathrm{M} \mathrm{Na}$ phosphate buffer $(\mathrm{pH}$ 6.86). Because of their sensitivity to light (in the blue region of the spectrum), the drugs were weighed and their solutions were prepared in a darkened room. All the studied solutions were prepared using twice-distilled water immediately before the measurements.

In the self-association study, aqueous solutions of riboflavin in the concentration range $2.5 \cdot 10^{-3}$ to $5 \cdot 10^{-5} \mathrm{M}$ were obtained by the method of successive dilution. When recording the spectra, we used glass cuvets with optical path length $10.0,5.0100,2.0082,1.0202,0.5062,0.2020,0.1065,0.0505$, and $0.0207 \mathrm{~cm}$. While recording the spectra, the temperature inside the cuvet compartment of the spectrophotometer $(298 \mathrm{~K})$ was held constant within $\pm 0.25 \mathrm{~K}$ by a thermostat device.

In the heteroassociation study of the systems RFN-NAS and RFN-caffeine, the riboflavin $(0.1 \mathrm{M}$ Na phosphate buffer, $\mathrm{pH}$ 6.86) was titrated by sodium salicylate in the concentration range $2 \cdot 10^{-4}$ to $4 \cdot 10^{-1} \mathrm{M}$ and by caffeine in the range $8 \cdot 10^{-4}$ to $6 \cdot 10^{-1} \mathrm{M}$. The riboflavin concentration was held constant in the mixed solutions $\left(C_{\mathrm{RFN}}=\left[B_{0}\right]\right.$ $=5 \cdot 10^{-5} \mathrm{M}=$ const $)$. The effect of urea on formation of heteroassociates was studied for a constant urea concentration $(1.0 \mathrm{M})$ in the solutions. The measurements were made in quartz cuvets with optical path length $1.02 \mathrm{~cm}$ at constant temperature $T=298 \pm 0.25 \mathrm{~K}$.

Results and Discussion. Self-association of riboflavin. Figure 2 shows the experimental spectra of aqueous solutions of riboflavin $(0.1 \mathrm{M} \mathrm{Na}$ phosphate buffer, $\mathrm{pH} 6.86, T=298 \mathrm{~K})$, measured at riboflavin concentrations in the range $5 \cdot 10^{-5}$ to $2.5 \cdot 10^{-3} \mathrm{M}$. As we see, when the riboflavin concentration is increased in aqueous solution, the absorbance decreases at the wavelength of the monomer absorption maximum $\left(\lambda_{\max }=446 \mathrm{~nm}\right)$ and at the same time an isosbestic point appears at the wavelength $\lambda \approx 490 \mathrm{~nm}$. The spectral changes and the presence of an isosbestic point suggest the existence in solution of an equilibrium state between the monomer and dimer forms of the riboflavin molecules.

The dynamic equilibrium in solution was modeled by the scheme [37]:

$$
B_{1}+B_{1} \stackrel{K_{\mathrm{d} B}}{\longrightarrow} B_{2}
$$

where $K_{\mathrm{d} B}$ is the equilibrium dimerization constant of the molecules; $B_{1}$ and $B_{2}$ are the monomers and dimers of the riboflavin molecules. The total concentration of molecules in solution is:

$$
\left[B_{0}\right]=\left[B_{1}\right]+2\left[B_{2}\right],
$$

where $\left[B_{0}\right]$ is the initial concentration of the substance; $\left[B_{1}\right]$ is the monomer concentration; $\left[B_{2}\right]=K_{\mathrm{d} B}\left[B_{1}\right]^{2}$ is the dimer concentration.

The overall molar absorption coefficient $\varepsilon$, provided by the monomer and dimer components of the riboflavin molecules, can be represented in the form: 


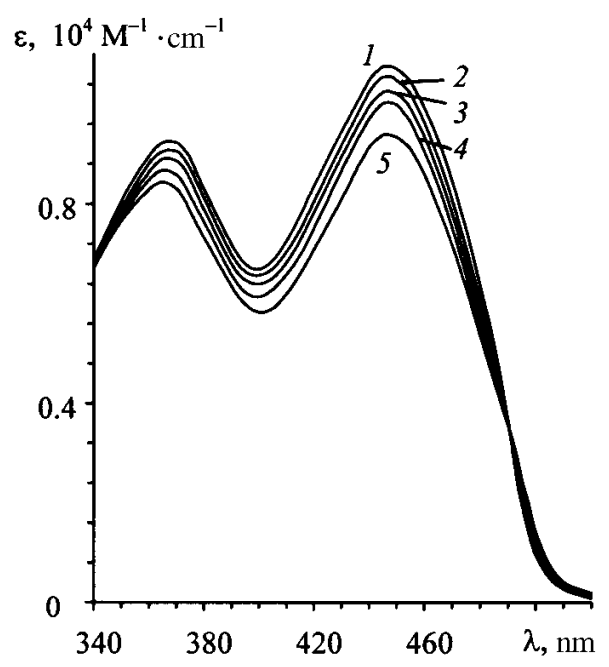

Fig. 2 Electronic absorption spectra of aqueous solutions of riboflavin (RFN) $(0.1 \mathrm{M} \mathrm{Na}$ phosphate buffer, $\mathrm{pH} 6.86, T=298 \mathrm{~K}): C_{\mathrm{RFN}}=5 \cdot 10^{-5}(1)$, $2 \cdot 10^{-4}(2), 5 \cdot 10^{-4}(3), 1 \cdot 10^{-3}(4)$ and $2.5 \cdot 10^{-3}(5) \mathrm{M}$.

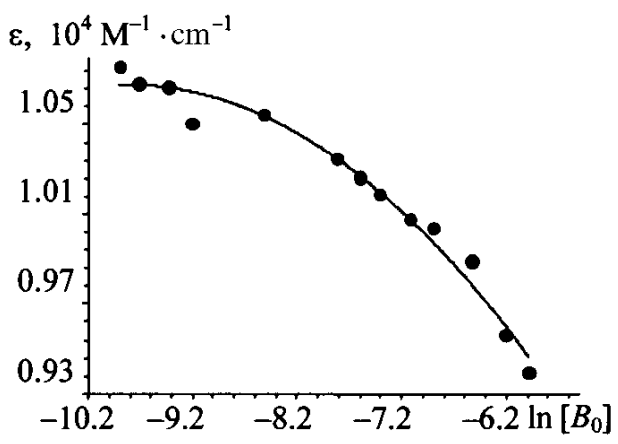

Fig. 3 Molar absorption coefficient for an aqueous solution of riboflavin $(0.1$ M Na phosphate buffer, $\mathrm{pH} 6.86, T=298 \mathrm{~K}$ ) at the wavelength of the riboflavin absorption maximum $\left(\lambda_{\max }=446 \mathrm{~nm}\right)$ vs. the logarithm of the concentration.

$$
\varepsilon=\varepsilon_{\mathrm{m}} f_{\mathrm{m}}+\varepsilon_{\mathrm{d}} f_{\mathrm{d}}
$$

where $\varepsilon_{\mathrm{m}}, \varepsilon_{\mathrm{d}}$ are the molar absorption coefficients of the monomers and the riboflavin molecules within the dimer complexes; $f_{\mathrm{m}}=\left[B_{1}\right] /\left[B_{0}\right]$ is the equilibrium mole fraction of the molecules in monomer form; $f_{\mathrm{d}}=2 K_{\mathrm{d} B}\left[B_{1}\right]^{2} /\left[B_{0}\right]$ is the equilibrium mole fraction of molecules within the dimers. The concentration $\left[B_{1}\right]$ is determined from the law of conservation of mass (2).

The extinction coefficients of the monomer and dimer forms of the riboflavin molecules $\left(\varepsilon_{\mathrm{m}}\right.$, $\left.\varepsilon_{\mathrm{d}}\right)$ and the equilibrium dimerization constant of the molecules $\left(K_{\mathrm{d} B}\right)$ are determined as the model parameters:

$$
\varepsilon=\varepsilon_{\mathrm{d}}+\left(\varepsilon_{\mathrm{d}}-\varepsilon_{\mathrm{m}}\right) \frac{1-\sqrt{1+8\left[B_{0}\right] K_{\mathrm{d} B}}}{4 K_{\mathrm{d} B}\left[B_{0}\right]}
$$

from the experimental concentration dependence at the wavelength $446 \mathrm{~nm}$ (Fig. 3).

As a result of the calculations, we obtained $K_{\mathrm{d} B}=125 \pm 40 \mathrm{M}^{-1}, \varepsilon_{\mathrm{m}}=(10.8 \pm 0.8) \cdot 10^{3} \mathrm{M}^{-1} \cdot \mathrm{cm}^{-1}, \varepsilon_{\mathrm{d}}=(6.5$ $\pm 1.1) \cdot 10^{3} \mathrm{M}^{-1} \cdot \mathrm{cm}^{-1}$. The value of the equilibrium dimerization constant for the riboflavin molecules $K_{\mathrm{d} B}$ is probably determined by the structure of the riboflavin molecule and the experimental conditions. 


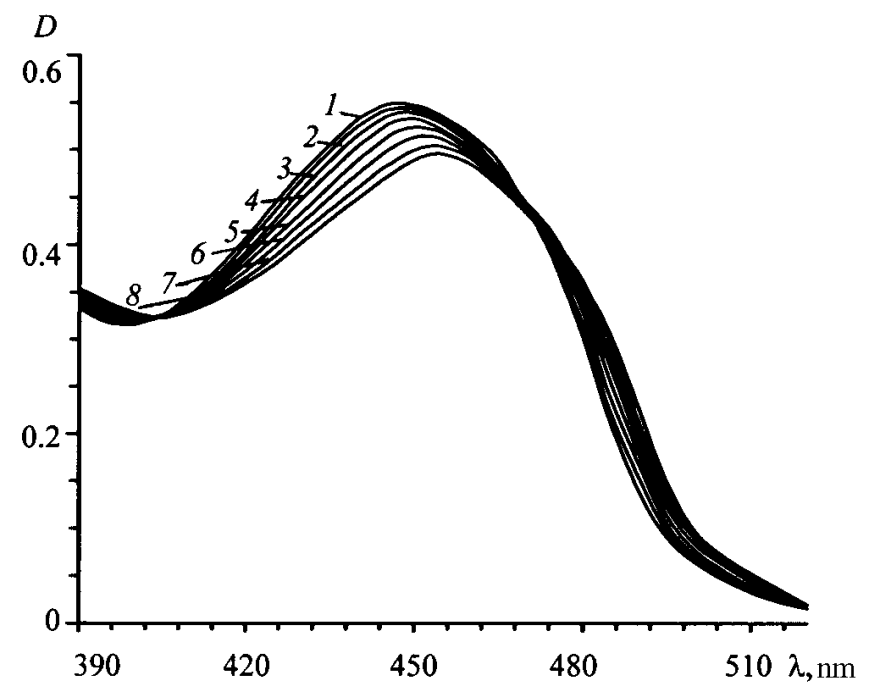

Fig. 4 Absorption spectra of aqueous solutions of heterocomplexes $(0.1 \mathrm{M} \mathrm{Na}$ phosphate buffer, $\mathrm{pH} 6.86, T=298 \mathrm{~K}$ ) of the system riboflavin-sodium salicylate $\left([\mathrm{RFN}]=5 \cdot 10^{-5} \mathrm{M}=\right.$ const) for NAS concentrations: 0 (1), $2 \cdot 10^{-4}$ (2), $1.5 \cdot 10^{-3}(3), 1.25 \cdot 10^{-2}(4), 3 \cdot 10^{-2}(5), 5 \cdot 10^{-2}(6), 1 \cdot 10^{-1}(7)$, and $2 \cdot 10^{-1}$ (8) $\mathrm{M}$.

Heteroassociation of riboflavin and NAS or caffeine. Figure 4 shows the electronic absorption spectra of an aqueous solution of riboflavin $\left(C_{\mathrm{RFN}}=\left[B_{0}\right]=5 \cdot 10^{-5} \mathrm{M}=\right.$ const $)$ when NAS is added to it at different concentrations $\left(2 \cdot 10^{-4}-4 \cdot 10^{-1} \mathrm{M}\right)$.

In titration of riboflavin by sodium salicylate, we observe changes in the electronic spectrum of riboflavin, expressed in the hypochromic effect and the bathochromic shift of the absorption bands relative to the maximum of the flavin monomer band $(446 \mathrm{~nm})$, and also in the appearance of isosbestic points at wavelengths of $405 \mathrm{~nm}$ and 470 $\mathrm{nm}$. These changes in the absorption spectra of flavin and also the presence of isosbestic points suggest the presence of specific interactions [38] between the riboflavin and NAS molecules, and formation of heterocomplexes of the interacting molecules in aqueous solution.

Heteroassociation of NAS (caffeine) and riboflavin molecules was analyzed using the Benesi-Hildebrand equation [39] for the condition $\left[A_{0}\right] \gg\left[B_{0}\right]$, where $\left[A_{0}\right]$ is the initial concentration of NAS (caffeine).

The equilibrium in solution was modeled by the scheme [37]:

$$
A_{1}+B_{1} \stackrel{K}{\longleftrightarrow} A_{1} B_{1}, A_{1}+A_{1} \stackrel{K_{\mathrm{d} A}}{\longrightarrow} A_{2},
$$

where $K$ is the equilibrium heteroassociation constant for the NAS (caffeine) and riboflavin molecules; $K_{\mathrm{d} A}$ is the equilibrium dimerization constant for the NAS (caffeine) molecules; $A_{1}$ and $A_{2}$ are the monomers and dimers of the NAS (caffeine) molecules); $A_{1} B_{1}$ is the heterocomplex.

The heteroassociation constant for the overall process of formation of the complex is determined by the equation:

$$
K=\frac{\left[A_{1} B_{1}\right]}{\left[A_{1}\right]\left(\left[B_{0}\right]-\left[A_{1} B_{1}\right]\right)},
$$

where $\left[A_{1} B_{1}\right]$ is the concentration of the heterocomplex; $\left[A_{1}\right]$ is the concentration of NAS (caffeine) monomers.

Eliminating the concentration of the complex $\left[A_{1} B_{1}\right]$ from formula (6), expressed in terms of its optical density using the Bouguer-Lambert-Beer law, we obtain:

$$
D_{k}=\frac{\varepsilon_{k} l\left[B_{0}\right] K\left[A_{1}\right]}{K\left[A_{1}\right]+1},
$$




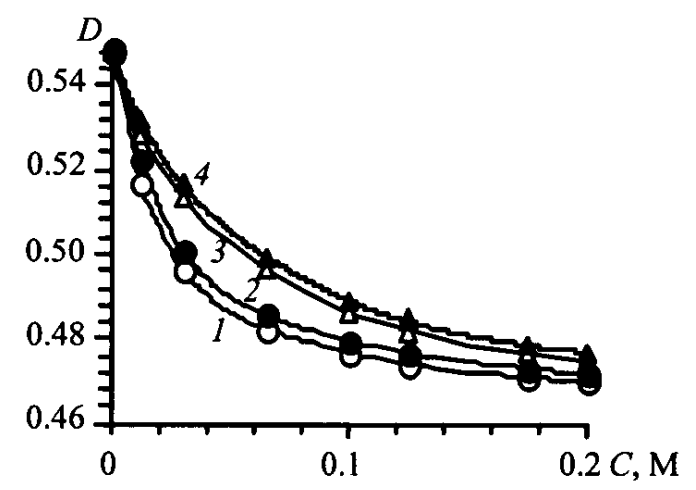

Fig. 5 Concentration dependences of the optical density of aqueous solutions (0.1 M Na phosphate buffer, pH 6.86; $T=298 \mathrm{~K}$ ) of the molecular complexes: RFN-caffeine (1); RFN-caffeine-urea (2); RFN-NAS (3); RFN-NASurea (4) $\left([\mathrm{RFN}]=5 \cdot 10^{-5} \mathrm{M}=\right.$ const; $[$ urea $]=1.0 \mathrm{M}=$ const $)$ at the wavelength of the riboflavin absorption maximum $\left(\lambda_{\max }=446 \mathrm{~nm}\right)$.

where $D_{k}$ and $\varepsilon_{k}$ are the optical density and the molar absorption coefficient of the riboflavin molecules within the heterocomplex at the wavelength corresponding to the absorption maximum of the riboflavin monomers: $l$ is the optical path length in the solution of the heterocomplex.

Transforming the optical density of the solution $D=\varepsilon_{\mathrm{m}} l\left[B_{1}\right]+\varepsilon_{k} l\left[A_{1} B_{1}\right]$ for the riboflavin molecules, taking into account Eq. (7), we obtain the concentration dependence of the overall absorption of the studied molecules:

$$
D=D_{\mathrm{m}} \frac{\Delta \varepsilon l\left[B_{0}\right] K\left[A_{1}\right]}{K\left[A_{1}\right]+1},
$$

where $D_{\mathrm{m}}$ is the optical density of the dilute solution containing only riboflavin; $\left[B_{0}\right]=5 \cdot 10^{-5} \mathrm{M} ; \Delta \varepsilon=\varepsilon_{\mathrm{m}}-\varepsilon_{k} ;\left[A_{1}\right]$ $=\left(-1+\sqrt{1+8 K_{\mathrm{d} A}\left[A_{0}\right]}\right) / 4 K_{\mathrm{d} A}$ (the formula for calculating the concentration of NAS (caffeine) monomers) is obtained from an equation analogous to Eq. (2)).

Expression (8), describing the optical density of the solution, contains three unknown parameters: $K, K_{\mathrm{d} A}$, and $\Delta \varepsilon$, determined from the concentration dependences of riboflavin absorption at the wavelength $446 \mathrm{~nm}$ (Fig. 5).

Using the modified Benesi-Hildebrand equation (7), we obtained the equilibrium constants for the formation of RFN-NAS (caffeine) heterocomplexes and dimer complexes of NAS (caffeine) for riboflavin and NAS: $K=25 \pm$ $4 \mathrm{M}^{-1}$ and $K_{\mathrm{d} A}=2.7 \pm 0.5 \mathrm{M}^{-1}$, and for riboflavin and caffeine: $K=74 \pm 11 \mathrm{M}^{-1}$ and $K_{\mathrm{d} A}=17 \pm 1.5 \mathrm{M}^{-1}$. The dimerization and heteroassociation constants vary in the following order: $K_{\mathrm{d} A}<K<K_{\mathrm{d} B}$.

The intermediate value of the heteroassociation constant in both the first and the second case $\left(K_{\mathrm{d} A}<K<\right.$ $\left.K_{\mathrm{d} B}\right)$ is due to the electronic configuration of the complexes formed. We should point out that the heteroassociation constants of the studied molecular systems take on an intermediate value between the equilibrium self-association constants for caffeine, NAS, and riboflavin, as has also been observed for other molecular systems [40]. The smallest value of $K_{\mathrm{d} A}$ is obtained for the NAS molecules. This is possibly connected with the structure of NAS, containing one aromatic ring, and with the weak dispersion forces stabilizing the dimer $\pi$ complexes. The constant $K_{\mathrm{d} B}$ is mainly determined by the presence in the riboflavin molecule of a hydrophobic ring system with a large dipole moment, promoting dimerization of the molecules, and a massive ribityl chain, creating steric hindrances in formation of the dimers.

The closeness of the chemical structures of riboflavin and flavin mononucleotide allows us to hypothesize that the nature of their interaction in self-association and heteroassociation is similar. Like many aromatic dyes and antibiotics, flavin mononucleotide molecules form stacked associates as a result of vertical interaction between chromophores (stacking) [41-44]. Based on the spectrophotometric studies and exciton theory, in [45] the structure parameters of the flavin mononucleotide dimer in aqueous solution were established and a sandwich (plane-parallel) dimer model was proposed. 
The equilibrium constants for the formation of RFN-NAS and RFN-caffeine heterocomplexes in the presence of urea in solution are $K=17 \pm 3 \mathrm{M}^{-1}$ and $K=53 \pm 7 \mathrm{M}^{-1}$ respectively, i.e., the stability of the complexes decreases.

Conclusion. We have shown that riboflavin effectively aggregates in solution and also forms complexes with sodium salicylate and caffeine. Self-associates and heteroassociates of the molecules are predominantly stabilized by dispersion and hydrophobic interactions between the aromatic chromophores in the complexes. Analysis of the dynamic equilibrium of the interacting molecules in solution allowed us to conclude that heteroassociation with caffeine and sodium salicylate reduces the monomer concentration of riboflavin molecules in solution. The effect of urea on the studied molecular systems is apparent in the decrease in the binding forces in the heterocomplexes due to weakening of the hydrophobic effect. Considering that the major biological effect of riboflavin is exhibited in the interaction between its monomers and molecular receptors of the cell, we can hypothesize that RFN-caffeine and RFN-NAS heteroassociation should lead to a change in the biological activity of riboflavin in the presence of caffeine and NAS.

\section{REFERENCES}

1. E. Chu and V. T. De Vita, Physicians' Cancer Chemotherapy Drug Manual, Jones and Bartlett Publ., London (2003).

2. M. Menozzi, L. Valentini, E. Vannini, and F. Arcamone, J. Pharm. Sci., 73, 766-770 (1984).

3. J. Piosik, M. Zdunek, and J. Kapuscinski, Biochem. Pharm., 63, 635-646 (2002).

4. E. D. Kharash and R. Novak, Arch. Biochem. Biophys., 212, 20-36 (1981).

5. A. Ramu, M. M. Mehta, and J. Liu, Cancer Chem. Pharm., 46, 449-458 (2000).

6. R. P. Webster, M. D. Gawde, and R. K. Bhattacharya, Cancer Lett., 98, 129-135 (1996).

7. J. Pangrekar, K. Krishnaswamy, and V. Jagadeesan, Fd. Chem. Toxic., 31, 745-750 (1993).

8. R. W. Larsen, R. Jasuja, R. K. Hetzler, P. T. Muraoka, V. G. Andrada, and D. M. Jameson, Biophys. J., 70, 443-452 (1996).

9. J. W. Jefferson, J. Clin. Psych., 59, 31-37 (1998).

10. V. Massey, Biochem. Soc. Trans., 28, 283-296 (2000).

11. W. Shuping, J. Zhiquin, L. Heting, L. Yang, and Z. Daixun, Molecules, 6, 52-60 (2001).

12. R. Goodrich, Vox Sanguinis, 78, Suppl. 1, 211-215 (2000).

13. C. V. Hanson, in: Medical Virology, Elsevier, Amsterdam (1983), Vol. 2, pp. 45-79.

14. C. H. Chiao and Z. Z. Liu, Za Zhi, 11, 92-98 (1989).

15. R. S. Rivlin, Exp. Med. Biol., 206, 349-355 (1986).

16. J. Piosik, K. Ulanowska, A. G. Wisniewska, A. Czy, J. Kapuscinski, and G. Wegryzn, Mutation Res., 530, 47-57 (2003).

17. B. K. Jacobsen, E. Bjelke, G. Kvale, and I. Heuch, J. Natl. Cancer Inst., 76, 823-831 (1986)

18. L. Rosenberg, M. M. Werler, J. R. Palmer, D. W Kaufman, M. E. Warshauer, P. D. Stolley, and S. Shapiro, Am. J. Epidemiol., 130, 895-903 (1989).

19. C. La Vecchia, Cancer Res., 49, 1049-1051 (1989).

20. C. La Vecchia, M. Ferraroni, E. Negri, B. D’Avanzo, A. Decarli, F. Levi, and S. Franceschi, Int. J. Cancer, 40, 469-473 (1987).

21. L. J. Vatten, K. Solvoll, and E. B. Loken, Br. J. Cancer, 62, 267-270 (1990).

22. W. A. Zatonski, P. Boyle, K. Przewozniak, P. Maisonneuve, K. Drosik, and A. M. Walker, Int. J. Cancer, 53, 601-607 (1993).

23. A. Linos, D. A. Linos, N. Vgotza, A. Souvatzoglou, and D. A. Koutras, Acta Chir. Scand., 155, 317-320 (1989).

24. A. Wallqvist, D. G. Covell, and D. Thirumalai, J. Am. Chem. Soc., 120, $427-433$ (1998).

25. A. Caballero-Herrera, K. Nordstrand, K. D. Berndt, and L. Nilsson, Biophys. J., 89, $842-857$ (2005).

26. H. S. Frank and F. J. Franks, J. Chem. Phys., 48, 4746-4752 (1968).

27. C. Tanford, Hydrophobic Effect: Formation of Micelles and Biological Membranes, Wiley, New York (1979).

28. D. Balasubramanian and S. E. Friberg, in: E. Matijevic, ed., Surface and Colloid Science, Plenum Press, New York (1993), Vol. 15.

29. P. Jarho, A. Urtti, D. W. Pate, P. Suhonen, and U. Jarvinen, Int. J. Pharm., 137, 209-216 (1996). 
30. M. A. Etman and A. H. Nada, Acta Pharm., 49, 291-298 (1999).

31. I. A. Darwish, A. T. Florence, and A. M. Saleh, J. Pharm. Sci., 78, 577-581 (1989).

32. E. Touitou, F. Alhaique, P. Fisher, A. Memoli, F. M. Riccieri, and E. Santucci, J. Pharm. Sci., 76, 791-793 (1987).

33. R. E. Coffman and D. O. Kildsig, J. Pharm. Sci., 85, 951-954 (1996).

34. N. K. Jain, V. V. Patel, and L. N. Taneja, Pharmacia, 43, 194-196 (1988).

35. T. Loftsson, H. Fridriksdottir, A. M. Sigurdardottir, and H. Ueda, Int. J. Pharm., 110, 169-177 (1994).

36. B. K. Roy and S. P. Moulik, Current Sci., 85, 1147-1155 (2003).

37. S. F. Baranovskii, P. A. Bolotin, and M. P. Evstigneev, Zh. Prikl. Spektr., 73, 158-163 (2006).

38. S. K. Gorbatsevich, Spectroscopy of Intermolecular Interactions. Nonlinear Effects [in Russian], Bel. Gos. Univ., Minsk (2002), p. 150.

39. H. A. Benesi and J. H. Hildebrand, J. Am. Chem. Soc., 71, $2703-2707$ (1949).

40. D. B. Davies, D. A. Veselkov, L. N. Djimant, and A. N. Veselkov, Eur. Biophys. J., 30, 354-361 (2001).

41. M. Kainosho and Y. Kyogoku, Biochemistry, 11, 741-752 (1972).

42. S. Shinkhai, A. Harada, Y. Ishikawa, O. Manabe, and F. Yoneda, Chem. Lett., 479-482 (1981).

43. M. F. Zipplies and M. A. Staab, Tetrahedron Lett., 25, 1035-1038 (1984).

44. Y. Yano, E. Ohya, and Y. Kuwabara, Chem. Lett., 1009-1012 (1984).

45. H. Grajek, G. Zurkowska, R. Drabent, and C. Bojarski, Biochim. Biophys. Acta, 881, 241-247 (1986). 